\title{
Comparison of the short-term postoperative results of prone positioning and lateral decubitus positioning during thoracoscopic esophagectomy
}

\author{
Nai Liang Li $^{1}$, Wen-Ling Peng ${ }^{1}$, Chia-Chuan Liu², Chih-Hsun Shih ${ }^{2}$ \\ ${ }^{1}$ Department of Anesthesiology, Koo Foundation Sun Yat-Sen Cancer Center, Taipei, Taiwan \\ ${ }^{2}$ Department of Surgery, Koo Foundation Sun Yat-Sen Cancer Center, Taipei, Taiwan
}

Videosurgery Miniinv 2015; 10 (1): 37-43

DOI: $10.5114 /$ wiitm.2015.48698

\begin{abstract}
Introduction: The conventional approach during thoracoscopic esophagectomy was performed in the left lateral decubitus position (LLDP). Recently, thoracoscopic esophagectomy in the prone position (PP) has attracted the attention of surgeons.

Aim: To report institutional experience with thoracoscopic esophagectomy in PP and compare it with the conventional LLDP approach.

Material and methods: We reviewed 59 consecutive patients who had presented with esophageal cancer undergoing three-stage thoracoscopic/laparoscopic esophagectomy (TLE) from May 2011 to Dec 2013. The TLE was sequentially performed on enrolled patients in LLDP from May 2011 to Oct 2012 and in PP from Nov 2012 to Dec 2013. Immediate postoperative outcomes were collected and compared to determine differences between the 2 groups.

Results: Thirty-eight patients had their operations in LLDP and 21 in PP. No differences in blood loss, respiratory condition during surgery, or postoperative pain scores were observed between the 2 groups. The PP had a shorter thoracic stage duration (3.4 vs. $3.9 \mathrm{~h} ; \mathrm{p}=0.03$ ) and shorter intensive care unit (ICU) stay (1.0 vs. 1.5 days; $p=0.03$ ) but yielded a similar number of lymph nodes. Incidence of complications was similar between the 2 groups, except significantly lowerincidence of pneumonia in PP (0\% vs. $21.1 \%$; $p=0.04)$ and higher incidence of hoarseness in PP $(52.4 \%$ vs. $23.7 \%$; $p=0.03)$. The symptoms resolved within 3 months in all patients except in the 2 patients with vocal cord palsy.

Conclusions: It is feasible and safe to perform thoracoscopic esophagectomy by adopting the prone position. Thoracoscopic esophagectomy in the prone position is potentially associated with fewer major complications and shorter ICU stay.
\end{abstract}

Key words: thoracoscopic esophagectomy, prone position, esophageal cancer, minimally invasive esophagectomy.

\section{Introduction}

With the evolution of surgical techniques, screening of early disease, neoadjuvant therapy, and intensive care, there has been significant improvement of the survival rate of esophageal cancer during the past few decades [1, 2]. Nonetheless, esophagectomy is still a major operation associated with signif- icant morbidity and mortality $[3,4]$. In an attempt to reduce invasiveness and postoperative morbidity, and to shorten the recovery time, minimally invasive esophagectomy (MIE) has been increasingly applied in treating esophageal cancer [5]. A variety of minimally invasive procedures have been described, including transhiatal laparoscopic esophagectomy, the

\section{Address for correspondence}

Nai Liang Li, Department of Anesthesiology, Koo Foundation Sun Yat-Sen Cancer Center, 125, Lih-Der Road, 11259 Taipei, Taiwan, phone: 886-2-28970011, e-mail: Inl@kfsyscc.org 
thoracoscopic/laparoscopic two-stage (Ivor Lewis) approach, and the thoracoscopic/laparoscopic threestage (cervical, mediastinal, and abdominal) McKeown approach.

Thoracoscopic mobilization of the esophagus during the three-stage thoracoscopic/laparoscopic esophagectomy (TLE) was originally performed in the left lateral decubitus position (LLDP) in the early 1990s [6]. The feasibility and minimal invasiveness of this procedure compared to open surgery have been demonstrated [7]. However, a low rate of adopting this procedure, which requires meticulous technique to maintain the surgery field, was also noted [8]. Recently, prone thoracoscopic esophagectomy has been increasingly adopted for TLE, with the advantages of esophagus mobilization, excellent exposure of the operative field, and better ergonomics [9-11]. Although the prone position (PP) is thought to have theoretical physiological and ergonomic advantages for the patient and surgeon, there are still controversies in adopting the prone position during MIE.

The evolution of surgical techniques and anesthesia has also happened in our hospital in the past few years. PP has been employed since Nov 2012. The LLDP during TLE was routine practice before then. One-lung ventilation with a double-lumen endotracheal tube was employed during the thoracic stage in patients in LLDP before Nov 2012. Subsequently, single lumen endotracheal intubation with $\mathrm{CO}_{2}$ insufflation to collapse the right lung was applied during the thoracic stage in PP.

\section{Aim}

We would like to compare the immediate post-operative outcomes between two groups of patients receiving TLE under the two different positions.

\section{Material and methods}

\section{Patient enrollment}

We collected data of all consecutive patients receiving three-stage TLE in our hospital until Dec 2013. In our institute, three-stage TLE comprised procedures of en bloc thoracoscopic esophagectomy, intrathoracic radical lymphadenectomy followed by laparoscopic gastric mobilization, cardiectomy, formation of a gastric conduit, gastric tube pull-up via a post-mediastinal route, and cervical anastomosis. These procedures were performed for stage I-IV esophageal cancer by two chest surgeons.
Between May 2011 and Dec 2013, 59 patients underwent three-stage TLE. The LLDP was sequentially employed in all patients undergoing threestage TLE from May 2011 to Oct 2012. The PP was subsequently employed in all patients from Nov 2012 in our institution.

\section{Anesthesia, ventilation strategy, and postoperative pain control}

Heart rate (by electrocardiography), $\mathrm{CO}_{2}$ production (by capnography), arterial blood pressure, central venous pressure, blood oxygen saturation (by pulse oximetry), and nasal temperature were monitored. Lactated Ringer's solution was infused at $20 \mathrm{ml} / \mathrm{kg} / \mathrm{h}$ during the induction of anesthesia and to maintain preoperative central venous pressure. Hypotension (arterial blood pressure < 90 mm Hg) was corrected by increasing the rate of intravenous fluid infusion and by administration of ephedrine. Blood was replaced as necessary. The hemoglobin level was maintained at more than $8.0 \mathrm{~g} / \mathrm{dl}$ with transfusion of packed red blood cells. Nasal temperature was maintained between $36^{\circ} \mathrm{C}$ and $37^{\circ} \mathrm{C}$ using a warming device (Bair Hugger; Augustine Medical Inc., Minneapolis, MN).

To obtain chest analgesia and ameliorate pain during the postoperative respiratory maneuver, the epidural or paravertebral space catheterization was implanted at the T6-8 level at the operating table prior to the induction of anesthesia. Rescue analgesia for the abdominal and neck wound with intravenous morphine $0.1 \mathrm{mg} / \mathrm{kg}$ was administered if the pain level was beyond moderate.

All patients received general anesthesia. The standardized anesthetic technique with general anesthetics consisted of propofol 2 to $2.5 \mathrm{mg} / \mathrm{kg}$, cisatracurium $0.15 \mathrm{mg} / \mathrm{kg}$, and fentanyl $100 \mu \mathrm{g}$ for endotracheal intubation, and $5 \%$ to $8 \%$ desflurane titrated according to age, blood pressure, and heart rate for maintenance. One-lung ventilation with left-sided double-lumen endotracheal tubes was employed during the thoracic stage in all patients in the LLDP group. After the thoracic stage was finished, the double-lumen endotracheal tubes were replaced with single lumen endotracheal tubes before commencement of the abdominal stage. In the PP group, patients were ventilated with single lumen endotracheal tubes throughout the three stages of the operation. During the thoracic stage, $\mathrm{CO}_{2}$ insufflation at $6 \mathrm{~mm} \mathrm{Hg}$ to collapse the right lung was 
performed in the PP group. The ventilator parameters for both groups were as follows: tidal volumes of no more than $6 \mathrm{ml} / \mathrm{kg}$ of predicted body weight during the thoracic stage if $\mathrm{O}_{2}$ saturation was over $90 \%$, no more than $8 \mathrm{ml} / \mathrm{kg}$ of predicted body weight during the remaining stages of the surgery, and positive end-expiratory pressure (PEEP) of $4 \mathrm{~mm} \mathrm{Hg}$ to $6 \mathrm{~mm} \mathrm{Hg}$. Recruitment maneuvers were performed in both groups at the end of the thoracic stage. Anesthesiologists were allowed to change the ventilation protocol at any point at the surgeon's request or if there was any concern about patient safety. After surgery, patients in both groups were extubated and transferred to the intensive care unit (ICU). Patients were discharged from the ICU when they met the discharge criteria including stable hemodynamics, absence of significant blood loss, and sufficient respiration under room air.

\section{Surgical procedures}

\section{Thoracic stage in prone position}

Patients were placed in a prone position with the right arm raised above the head and left arm kept at the left side of the body. Four thoracostomies were created. One $10 \mathrm{~mm}$ port was placed at the ninth intercostal space (ICS) along the posterior axillary line. Another $10 \mathrm{~mm}$ port was placed at the seventh ICS along the mid-axillary line. Two $5 \mathrm{~mm}$ ports were placed at the fourth ICS and sixth ICS along the posterior axillary line and just inferior to the scapular tip, respectively. Lymph nodes along the right recurrent laryngeal nerve and supra-diaphragmal lymph nodes were dissected. The thoracic duct was identified and carefully preserved. The esophagus and the surrounding peri-esophageal and sub-carinal lymph nodes were mobilized and dissected en bloc. Lymph nodes along the left recurrent laryngeal nerve were dissected, with the nerve being carefully preserved.

\section{Thoracic stage in left lateral decubitus position}

The surgery procedure of TLE in LLDP has been described in our previous article [12]. After the patient was anesthetized, he or she was placed in LLDP. Four thoracoscopic ports were placed. The 1.2$\mathrm{cm}$ camera thoracoscopic port was placed through the right eighth ICS along the posterior-axillary line. The 1.2-cm working thoracoscopic port was placed at the right sixth ICS along the anterior axillary line. Two additional $0.5-\mathrm{cm}$ thoracoscopic ports were lo- cated at the right fourth ICS, one at the posterior axillary line and the other at the tip of the scapula. The operative procedure was similar to that in the prone group.

\section{Abdominal and cervical stage}

The abdominal and cervical stages were the same in the two groups. After the thoracic stage was finished, the patients were placed in the supine position. The sites for trocar placement were located at the upper abdomen, including one $12-\mathrm{mm}$ and one $5-\mathrm{mm}$ trocar over the bilateral paramedian about $5 \mathrm{~cm}$ above the umbilicus, another two $5-\mathrm{mm}$ trocars over the bilateral subcostal areas and another 12-mm trocar over the umbilicus, and a 5-mm trocar over the sub-xyphoid area. Gastric mobilization, abdominal regional lymphadenectomy, gastric tube formation, and jejunostomy were performed laparoscopically. The gastric tube was pulled up via a posterior mediastinal route. Anastomosis between the cervical esophagus and gastric conduit was performed.

\section{Statistical analysis}

Normally distributed data were presented as mean \pm standard deviation. Non-Gaussian data were presented as median ( $25^{\text {th }}$ percentile and $75^{\text {th }}$ percentile). Demographic data and surgical outcomes were analyzed using the $t$ test for continuous variables and Fisher's exact test for categorical variables. A two-tailed $p$ value of less than 0.05 was considered significant. All of the analyses were carried out using SAS 9.1 software (SAS Institute INC, Cary, NC, USA).

\section{Results}

A total of 59 consecutive esophageal cancer patients were enrolled; 38 constituted the LLDP group and 21 the PP group. Patient characteristics are shown in Table I. No significant difference was found between the 2 groups in baseline characteristics including age, sex, body mass index, American Society of Anesthesiologists (ASA) classification, smoking status, preoperative comorbidities, pulmonary function, tumor location, histological type, TNM stage, and pain control modality.

Operative parameters are shown in Table II. Conversion from thoracoscopic surgery to open surgery was not required in any of the patients. The number of thoracic lymph nodes harvested was similar between the two groups. There were no differences 
Table I. Baseline characteristics $(n=59)$

\begin{tabular}{|c|c|c|c|}
\hline Parameter & $\operatorname{LLDP}(n=38)$ & $\mathrm{PP}(n=21)$ & Value of $p$ \\
\hline Age, mean \pm SD [years] & $56.1 \pm 9.6$ & $55.6 \pm 9.4$ & 0.83 \\
\hline Gender, male/female, $n$ & $35 / 3$ & $19 / 2$ & 1.00 \\
\hline BW, mean \pm SD $[\mathrm{kg}]$ & $61.8 \pm 8.9$ & $65.2 \pm 11.3$ & 0.21 \\
\hline $\mathrm{BH}$, mean $\pm \mathrm{SD}[\mathrm{cm}]$ & $166.4 \pm 6.8$ & $168.2 \pm 7.2$ & 0.34 \\
\hline ASA (II/III/IV), $n$ & $17 / 20 / 1$ & $7 / 14 / 0$ & 0.21 \\
\hline Nonsmoking or quit > 3 months, $n$ (\%) & $19(50)$ & $11(52.4)$ & 0.86 \\
\hline Clinical stage (AJCC $6^{\text {th }}$ edition) I/II/III/IV/V, $n$ & $8 / 7 / 19 / 1 / 3$ & $2 / 17 / 12 / 0 / 0$ & 0.38 \\
\hline Preop CCRT (yes/no), $n$ & $25 / 13$ & $14 / 7$ & 0.95 \\
\hline Cell type (SCC/Ad-ca), $n$ & $36 / 2$ & $21 / 0$ & 0.53 \\
\hline Location, $\mathrm{u} / \mathrm{m} / \mathrm{l}, n$ & 8/17/13 & $2 / 14 / 5$ & 0.25 \\
\hline \multicolumn{4}{|l|}{ Preoperative PFT: } \\
\hline FVC & $101.2 \pm 16.9$ & $101.8 \pm 14.7$ & 0.88 \\
\hline $\mathrm{FEV}_{1}$ & $93.6 \pm 15.8$ & $95.5 \pm 13.9$ & 0.65 \\
\hline DLCO & $95.8 \pm 20.7$ & $91.6 \pm 19.7$ & 0.45 \\
\hline \multicolumn{4}{|l|}{ Preoperative conditions/comorbidities: } \\
\hline Anemia, $n(\%)$ & $26(68.4)$ & $13(61.9)$ & 0.61 \\
\hline $\mathrm{DM}, n(\%)$ & $5(13.2)$ & $1(4.8)$ & 0.41 \\
\hline Cirrhosis, $n(\%)$ & $3(7.9)$ & $2(9.5)$ & 0.75 \\
\hline $\mathrm{Hb}$ mean $\pm \mathrm{SD}[\mathrm{g} / \mathrm{dl}]$ & $12.7 \pm 1.6$ & $12.7 \pm 1.9$ & 0.94 \\
\hline Albumin, mean \pm SD [mg\%] & $4.2 \pm 0.3$ & $4.2 \pm 0.3$ & 0.99 \\
\hline Pain control modality: & & & 0.45 \\
\hline El/contPVB, $n(\%)$ & $37(97.4)$ & $21(100)$ & \\
\hline PCA, $n(\%)$ & $1(2.6)$ & $0(0)$ & \\
\hline
\end{tabular}

BW - body weight, $B H$ - body height, ASA - American Society of Anesthesiologists classification, Preop CCRT - preoperative chemoradiotherapy, SCC - squamous cell carcinoma, Ad-ca-adenocarcinoma, u/m/l-upper/middle/lower, preoperative PFT - preoperative pulmonary function test, El/contPVB - epidural infusion or continuous paravertebral block, PCA - patient-controlled analgesia, AJCC - American Joint Committee on Cancer, FVC - forced vital capacity, $F E V_{1}$ - forced expiratory volume in the first second, DLCO - diffusing capacity of the lungs for carbon monoxide, DM - diabetes mellitus, Hb-hemoglobin.

in blood loss, respiratory condition during surgery, or postoperative pain scores. However, when compared with patients in the LLDP group, those in the PP group had a shorter thoracic stage duration (3.4 vs. $3.9 \mathrm{~h} ; p=0.03$ ) and shorter ICU stay (1.0 vs. 1.5 days; $p=0.03)$.

Postoperative major morbidity occurred in $63.2 \%$ of patients in the LLDP group and $19.0 \%$ in the PP group. Minor morbidity occurred in $47.4 \%$ of patients in the LLDP group and $76.2 \%$ in the PP group. Rates of each single complication were similar between the 2 groups, except for pneumonia, which developed in a significantly smaller number of pa- tients in PP (0\% vs. 21.1\%; $p=0.04)$ and hoarseness in a significantly larger number of patients in PP (52.4\% vs. $23.7 \%$; $p=0.03$ ) (Table III). All the patients with hoarseness were examined with a bronchoscope. The symptom resolved within 3 months in all patients excepted in the 2 patients with vocal cord palsy. No operative deaths and no hospital deaths occurred in this series.

\section{Discussion}

Esophagectomy is a major procedure associated with a significant rate of morbidity $[3,4]$. Among 
Table II. Operative parameters

\begin{tabular}{|c|c|c|c|}
\hline Parameter & $\operatorname{LLDP}(n=38)$ & $\mathrm{PP}(n=21)$ & Value of $p$ \\
\hline Mean airway pressure, mean $\pm \mathrm{SD}\left[\mathrm{cm} \mathrm{H}_{2} \mathrm{O}\right]$ & $24.1 \pm 3.7$ & $23.9 \pm 3.1$ & 0.88 \\
\hline Intraop desaturation, yes/no, $n$ & $1 / 37$ & $0 / 21$ & 1.00 \\
\hline Low TV, $n(\%)$ & $18(47.4)$ & $16(76.2)$ & 0.03 \\
\hline Estimated blood loss, mean \pm SD [ml] & $104.9 \pm 93.3$ & $120.6 \pm 102.4$ & 0.55 \\
\hline Blood transfusion, $n$ (\%) & $6(15.8)$ & $5(23.8)$ & 0.50 \\
\hline Volume of colloid and PRBC, mean \pm SD [ml] & $784.2 \pm 458.3$ & $595.2 \pm 464.2$ & 0.14 \\
\hline Conversion to open thoracotomy, $n$ (\%) & $0(0)$ & $0(0)$ & 1.00 \\
\hline NO of LN dissected in thorax, mean \pm SD & $29.9 \pm 12.7$ & $27.2 \pm 11.9$ & 0.42 \\
\hline Thoracic operative time, mean \pm SD [h] & $3.9 \pm 0.7$ & $3.4 \pm 0.7$ & 0.03 \\
\hline Total operative duration, mean \pm SD [h] & $6.8 \pm 1$ & $7.0 \pm 1.1$ & 0.50 \\
\hline Ventilator dependence, mean \pm SD [days] & $0.2 \pm 1.0$ & $0.0 \pm 0.0$ & 0.46 \\
\hline ICU stay, mean \pm SD [days] & $1.5 \pm 1.4$ & $1.0 \pm 0$ & 0.03 \\
\hline Hospital stay, mean \pm SD [days] & $14.2 \pm 5.8$ & $12.6 \pm 3.2$ & 0.17 \\
\hline \multicolumn{4}{|l|}{ NRS pain score, median (Q1, Q3): } \\
\hline POD 0 & $5(3,8)$ & $3(0,6)$ & 0.10 \\
\hline POD 1 & $4.5(3,6)$ & $4(2,8)$ & 0.67 \\
\hline POD 2 & $4(2,6)$ & $3(2,5)$ & 0.53 \\
\hline POD 3 & $3(2,4)$ & $3(1,5)$ & 0.97 \\
\hline POD 4 & $2(1,3)$ & $2(2,3)$ & 0.53 \\
\hline
\end{tabular}

NO of $L N$ dissected in thorax - total number of lymph nodes harvested during the thoracic stage, Low TV-number of patients adopting tidal volume less than $6 \mathrm{ml} / \mathrm{kg}$ predicted body weight during thoracic stage, NRS - Numeric Rating Scale.

these major complications, pneumonia is the most feared. Postoperative pneumonia was defined as a positive bacterial culture of the sputum or abnormal chest radiograph with fever, or both in our study. In contrast to previous studies [13, 14] which showed that the incidence of pneumonia was similar in patients adopting the two different positions, we found that the incidence of pneumonia was significantly lower in the prone position group. When adopting the prone position, the advantages of better ergonomics and exposure of the operative field allowed lung retraction to be avoided and the thoracic stage to be shortened. Another potential advantage proposed by the previous study was the use of a single-lumen endotracheal tube to allow intermittent inflation of the right lung during the thoracic stage in the prone position. These factors reduced the traumatic insult to the patient's lungs, and this might ultimately lead to better postoperative respiratory functions.
Additionally, we controlled every preoperative and intraoperative risk factor [15] we know that may affect postoperative pulmonary complications, including age, body mass index, perioperative hemoglobin level, albumin level, ASA classification, smoking status, anesthetic protocol, blood transfusion, intraoperative parameters, and adequate postoperative analgesia. To prevent lung damage and postoperative pulmonary complications, we strictly adhered to the protective lung strategy [16] comprising low tidal volume, PEEP, and recruitment maneuvers. During the thoracic stage, the tidal volume would be set to be no larger than $6 \mathrm{ml} / \mathrm{kg}$ if it was possible to maintain $\mathrm{O}_{2}$ saturation at no less than $90 \%$. In the PP group, significantly more patients were able to keep their tidal volumes not larger than $6 \mathrm{ml} / \mathrm{kg}$ during the thoracic stage. This might also have contributed to the lower incidence of pneumonia in the PP group. 
Table III. Postoperative complications

\begin{tabular}{|lccc|}
\hline Complication & $\begin{array}{c}\text { LLDP } \\
(n=38)\end{array}$ & $\begin{array}{c}\text { PP } \\
(n=21)\end{array}$ & $\begin{array}{c}\text { Value } \\
\text { of } p\end{array}$ \\
\hline Minor: & $18(47.4 \%)$ & $16(76.2 \%)$ & \\
\hline Hoarseness & $9(23.7 \%)$ & $11(52.4 \%)$ & 0.03 \\
\hline Arrhythmia & $2(5.3 \%)$ & $2(9.5 \%)$ & 0.61 \\
\hline Fever & $1(2.6 \%)$ & $2(9.5 \%)$ & 1.00 \\
\hline Oozing of wound & $2(5.3 \%)$ & 0 & 0.53 \\
\hline Wound infection & $4(10.5 \%)$ & $1(4.8 \%)$ & 0.65 \\
\hline Major: & $24(63.2 \%)$ & $4(19.0 \%)$ & \\
\hline Vocal cord palsy & $1(2.6 \%)$ & $1(4.8 \%)$ & 1.00 \\
\hline Pneumonia & $8(21.1 \%)$ & 0 & 0.04 \\
\hline Pleural effusion & $1(2.6 \%)$ & $1(4.8 \%)$ & 1.00 \\
\hline Leakage & $9(23.7 \%)$ & $2(9.5 \%)$ & 0.30 \\
\hline Atelectasis & $3(7.9 \%)$ & 0 & 0.55 \\
\hline Tracheal laceration & $1(2.6 \%)$ & 0 & 1.00 \\
\hline TE fistula & $1(2.6 \%)$ & 0 & 1.00 \\
\hline
\end{tabular}

We found that the ICU stay was significantly shorter and the total major complication rate was significantly lower in the PP group, which might be attributed to the gravitational effect of this position and the consequent better exposure of the operative field and thus less tractional insult to the lungs.

Our experience was similar to a previous study [13] in that prone positioning provided better exposure of the operative field around the left recurrent laryngeal nerve and thus allowed for radical lymph node dissection around the left recurrent laryngeal nerve. Nonetheless, the numbers of lymph nodes harvested during the thoracic stage were similar in the 2 groups. Though the incidence of hoarseness was significantly higher in the prone group, rates of recurrent laryngeal nerve injury were not significantly different between prone and left lateral decubitus-positioned patients. The insult of the left recurrent laryngeal nerve might be minor and temporary. Long-term follow-up is necessary for the clinical relevance of the radical lymph node dissection along the left recurrent laryngeal nerve.

The conversion rate has been reported to be up to $7 \%$ in a previous study [17]. Although this procedure is technically demanding, there was no conversion from thoracoscopic surgery to open surgery in any of our patients.
There were some limitations of the study. First, it was a retrospective study using historical controls. Though identical perioperative protocols were applied throughout the period of the study, and more than 30 MIEs had been performed by each of the surgeons before 2011 when this study started, proficiency bias of the surgeons probably existed. As we know, the surgeon's experience is crucial to reduce morbidity in thoracoscopic esophagectomy [16]. The benefits of the relatively new approach in the prone position might be more evident as the sample size and surgeon's experience increase. Second, the small sample size of the study population may also have prevented exclusion of a type 2 error when comparing statistical differences between the two groups in some endpoints, such as each single postoperative complication.

\section{Conclusions}

In this study, we found that thoracoscopic esophagectomy with lung protective ventilation in the prone position is a technically feasible procedure. It produced better pulmonary outcomes compared to the lateral decubitus position. We also found that thoracoscopic mobilization of the esophagus in the prone position is an ergonomically better technique compared with that of the left lateral decubitus position. Comparative and randomized, multi-institutional studies are needed to validate the procedure, to reproduce the results, and to choose the best technique.

\section{Conflict of interest}

The authors declare no conflict of interest.

\section{References}

1. Portale G, Hagen JA, Peters JH, et al. Modern 5-year survival of resectable esophageal adenocarcinoma: single institution experience with 263 patients. J Am Coll Surg 2006; 202: 588-96.

2. Kim T, Grobmyer SR, Smith R, et al. Esophageal cancer - the five year survivors. J Surg Oncol 2011; 103: 179-83.

3. Chang AC, Ji H, Birkmeyer NJ, et al. Outcomes after transhiatal and transthoracic esophagectomy for cancer. Ann Thorac Surg 2008; 85: 424-9.

4. Connors RC, Reuben BC, Neumayer LA, et al. Comparing outcomes after transthoracic and transhiatal esophagectomy: a 5-year prospective cohort of 17,395 patients. J Am Coll Surg 2007; 205: 735-40.

5. Blencowe NS, Strong S, McNair AG, et al. Reporting of shortterm clinical outcomes after esophagectomy: a systematic review. Ann Surg 2012; 255: 658-66. 
6. Collard JM, Lengele B, Otte JB, et al. En bloc and standard esophagectomies by thoracoscopy. Ann Thorac Surg 1993; 56: 675-9.

7. Akaishi T, Kaneda I, Higuchi N, et al. Thoracoscopic en bloc total esophagectomy with radical mediastinal lymphadenectomy. J Thorac Cardiovasc Surg 1996; 112: 1533-40.

8. Committee for Scientific Affairs, Sakata R, Fujii Y, et al. Thoracic and cardiovascular surgery in Japan during 2009: annual report by the Japanese Association for Thoracic Surgery. Gen Thorac Cardiovasc Surg 2011; 59: 636-67.

9. Cuschieri A. Thoracoscopic subtotal oesophagectomy. Endosc Surg Allied Technol 1994; 2: 21-5.

10. Palanivelu C, Prakash A, Senthilkumar R, et al. Minimally invasive esophagectomy: thoracoscopic mobilization of the esophagus and mediastinal lymphadenectomy in prone position - experience of 130 patients. J Am Coll Surg 2006; 203: 7-16.

11. Fabian T, McKelvey AA, Kent MS, et al. Prone thoracoscopic esophageal mobilization for minimally invasive esophagectomy. Surg Endosc 2007; 21: 1667-70.

12. Li NL, Liu CC, Cheng SH, et al. Feasibility of combined paravertebral block and subcostal transversus abdominis plane block in postoperative pain control after minimally invasive esophagectomy. Acta Anaesthesiol Taiwan 2013; 51: 103-7.

13. Noshiro $\mathrm{H}$, Iwasaki $\mathrm{H}$, Kobayashi $\mathrm{K}$, et al. Lymphadenectomy along the left recurrent laryngeal nerve by a minimally invasive esophagectomy in the prone position for thoracic esophageal cancer. Surg Endosc 2010; 24: 2965-73.

14. Feng M, Shen Y, Wang H, et al. Thoracolaparoscopic esophagectomy: is the prone position a safe alternative to the decubitus position? J Am Coll Surg 2012; 214: 838-44.

15. Severgnini P, Selmo G, Lanza C, et al. Protective mechanical ventilation during general anesthesia for open abdominal surgery improves postoperative pulmonary function. Anesthesiology 2013; 118: 1307-21.

16. Osugi H, Takemura M, Higashino $M$, et al. Learning curve of video-assisted thoracoscopic esophagectomy and extensive lymphadenectomy for squamous cell cancer of the thoracic esophagus and results. Surg Endosc 2003; 17: 515-9.

17. Smithers BM, Gotley DC, McEwan D, et al. Thoracoscopic mobilization of the esophagus. A 6 year experience. Surg Endosc 2001; 15: 176-82.

Received: 10.11.2014, accepted: 8.01.2015 